\title{
El sistema de las profesiones y el profesional de la información
}

\author{
Miriam Vieira da Cunha \\ Profesora do Departamento de Ciência da Informação \\ Universidade Federal de Santa Catarina, Florianópolis, Brasil
}

\subsection{Resumen}

Reflexión sobre la evolución actual de las profesiones relacionadas con el tratamiento de la información dentro del sistema de las profesiones desde el enfoque de la Sociología de las Profesiones de Abbott y McGuire. Se enfatizan los cambios que están ocurriendo en el sistema de las profesiones en función del desarrollo de la sociedad del conocimiento. Como conclusión se resalta la necesidad de los profesionales de la información de repensar, en función de esta nueva realidad, su papel y sus relaciones con el mundo del trabajo para poder responder satisfactoriamente a las nuevas demandas de la sociedad.

Palabras clave: Profesional de la información. Sociología de las profesiones. Especialización profesional. Tendencias futuras.

\subsection{Abstract}

Reflection on the current evolution of the professions related with the information treatment in the frame of the system of professions from the point of view of the Sociology of Professions of Abbott and McGuire. The changes that are occurring in the system of professions as a consequence of the development of the knowledge society are analysed. It is concluded that a need exists that information professionals rethink from this new reality their role and their relationship with the labour market to respond to the new demands of the society.

Keywords: Information professional. Sociology of professions. Professional specialization. Future trends.

\section{Introducción}

Estamos viviendo, en el inicio de este siglo, un momento de transformaciones intensas en un contexto de cambios constantes en el ambiente tecnológico y organizacional. Estos cambios caracterizan la sociedad actual y el futuro de prácticamente todos los campos del quehacer humano. Sin duda, el adelanto de la

Scire. $9: 1$ (en.-jun. 2003) 85-93. 
tecnología constituye la principal base que fundamenta estos cambios. Es posible percibir, a través de estas transformaciones, la emergencia de nuevos métodos y relaciones de trabajo, de nuevas formas de organización empresarial, de nuevas propuestas educacionales y de nuevas formas de comunicación. Como consecuencia de esta realidad, se están desarrollando nuevas opciones profesionales, y se entrevén nuevas oportunidades de trabajo. Además de eso, nuevas estrategias de cooperación y una nueva arquitectura organizacional demandan competencias diferenciadas en los ambientes de trabajo, provocando la necesidad de nuevas formas de gestión de la información. Este ambiente, caracterizado por el crecimiento de la industria de la información y del conocimiento, impacta de forma singular en las organizaciones como consecuencia de nuevas demandas en un universo donde la única característica permanente es el cambio. En este sentido, este trabajo tiene como objetivo reflexionar sobre el sistema de las profesiones enfatizando las transformaciones que están ocurriendo en aquéllas que tienen como objetivo principal el trato con la información.

\section{Las características y la evolución de las profesiones}

Las profesiones están constituidas por grupos de personas que aplican conocimientos abstractos a casos particulares con el objetivo de resolver problemas específicos en favor de una clientela. Estos grupos pueden ser definidos como comunidades en el seno de las cuales sus miembros comparten una identidad, un compromiso personal, intereses específicos y una lealtad. La identidad personal se concreta a través del compartimiento de valores fundamentados en los servicios ofrecidos. Cada grupo profesional define sus reglas y las relaciones que establecen con la sociedad y con las demás profesiones. La alianza entre saber y servicio es reforzada porque estas reglas tienen un reconocimiento oficial.

De acuerdo con Hughes (citado por Bennet Jr \& Hokenstad Jr, 1973), el término "profesional" tiene su origen en la Edad Media, a partir del verbo "professare" que significaba realizar los votos religiosos. Esta connotación religiosa sugiere la idea de deber y de servicio prestado a clientes y la identificación del profesional con este servicio. Fue Flexer (citado Bennett \& Hokenstad, 1973) quien creó, en 1915, la primera definición sistematizada de lo que es una profesión. Para este autor, una profesión se fundamenta en una actividad intelectual, requiere de sus miembros la posesión de un conocimiento, tiene objetivos bien definidos, posee técnicas que pueden ser comunicadas y una organización propia, motivada por el deseo de trabajar por el bienestar de la sociedad.

Existen varias definiciones del concepto de profesional. Sin embargo, todas ellas cubren las nociones de monopolio de conocimientos, de autonomía y de ejecución de un servicio. El hecho de ejecutar servicios presupone una competencia en el dominio en cuestión y unos conocimientos específicos adquiridos a

Scire. $9: 1$ (en.-jun. 2003) 85-93. 
través de la formación. La formación garantiza el monopolio del conocimiento, da acceso a la calificación y al reconocimiento profesional, otorgando a estos profesionales el derecho a ofrecer servicios a la comunidad. La formación es organizada y generada por la profesión y constituye uno de los rasgos característicos de cada grupo profesional. Finalmente, la autonomía significa el derecho que los profesionales tienen a organizar y reglamentar sus actividades. Las organizaciones profesionales, a través de instrumentos como códigos de ética y normas de comportamiento, pretenden garantizar la calidad de los servicios ofrecidos. El código de ética está constituido por normas reconocidas por los miembros de la profesión que buscan mostrar su utilidad social, normalizar su actividad y reducir la competitividad interna. La orientación de servicio supone reglas implícitas de competencia y de performance.

De acuerdo con Matarazzo (citado por McGuire, 1993), todas las profesiones pasan por las mismas etapas de evolución. En un primer momento, se produce un ingreso desordenado de profesionales; a continuación, estos profesionales se organizan en corporaciones voluntarias. La siguiente etapa consiste en la imposición del requisito de formación. Esta exigencia es reforzada a través del reconocimiento de programas de enseñanza específicos. La última etapa se caracteriza por una mayor elaboración del proceso de reconocimiento profesional a través de la creación de especialidades.

La estructura cognitiva de las profesiones debe ser legitimada por la sociedad. En este sentido, cada profesión se relaciona con una comunidad a través de un contrato implícito. Este contrato le da el derecho exclusivo de ejercicio a cambio de la seguridad, calidad y eficacia de los servicios ofrecidos. Estos derechos proceden del conocimiento que legitima su campo de trabajo (Curry \& Wergin, 1993). Pueden incluir el monopolio de la práctica profesional, el control de la formación y del reclutamiento de sus miembros. Según Haug (1973), el monopolio del conocimiento especializado que una profesión ostenta asegura su lugar en la jerarquía del sistema profesional, su status y su prestigio. W. Moore (1970) precisa que, cuanto más sistematizados están los conocimientos de un campo de actividad, mayor es el monopolio sobre su espacio. Por tanto, el control del conocimiento se establece a partir de las relaciones que existen entre la práctica profesional y valores como legitimidad cultural, racionalidad y eficacia. Este autor añade también que el conocimiento común es indispensable para mantener la unión de un grupo profesional.

El conjunto de las profesiones forma un sistema. Abbott (1988) define este sistema como una estructura que relaciona las profesiones entre ellas de tal forma que la evolución de una afecta las otras. La evolución de este sistema depende de los ajustes que se realizan dentro de cada profesión. Estos ajustes son una consecuencia de la forma en que los diversos grupos profesionales controlan sus

Scire. $9: 1$ (en.-jun. 2003) 85-93. 
conocimientos y competencias. El control de la profesión es determinado por el dominio de las abstracciones que generan la práctica profesional. La habilidad de las profesiones en mantener su jurisdicción o su espacio, resulta, de esta forma, en parte, del prestigio de su sistema de conocimiento.

En su estudio sobre la evolución de las profesiones a través de sus interrelaciones, Abbott (1988) afirma que cada una de ellas ocupa un lugar específico en el sistema y desarrolla sus actividades en varios tipos de jurisdicciones. Este lugar se determina desde un núcleo central, un campo de actividades bien delimitado, sobre el cual el grupo ejerce un control completo y legal. A través de este control cada profesión procurará excluir a otros trabajadores de su campo. La fuerza y el éxito de una profesión resultan así legitimadas por la delimitación clara de su campo de competencia, por la delimitación de un espacio propio de acción y a través de su interacción con otras profesiones.

Existe reconocimiento de una especificidad cuando un grupo profesional dispone de un "savoir-faire" que no es compartido con otros grupos, otorgando así a cada uno de sus miembros un lugar específico en la sociedad. En este caso, el grupo tendrá el control total de un determinado espacio, como ocurre, por ejemplo, en el caso de la medicina. Algunas veces este control está subordinado a la intervención de otro grupo profesional, como en el caso de las profesiones paramédicas. Cuando los límites de una profesión no están claramente definidos, profesionales de otras áreas "invaden" su campo, produciéndose, de esta forma, una disputa por su espacio. Analizar el desarrollo de las profesiones es, en este sentido, analizar las conexiones existentes entre cada una de ellas y su campo de trabajo.

Cada profesión defiende intereses que le son propios. Sin embargo, en muchos casos, las características de una ocupación no son exclusivas de un grupo y derivan de especificidades de otras actividades. Además de esto, cuando la demanda hecha a un grupo profesional por el mercado de trabajo supera su capacidad de respuesta, este grupo pierde su espacio o se subdivide. Es lo que se produjo con las especialidades relacionadas con la Ingeniería, por ejemplo. La aparición de una nueva ocupación significa de esta forma que tiene actividades específicas suficientemente valoradas que se diferencian de otras y que tiene una utilidad reconocida públicamente. De esta forma, las especialidades profesionales aparecen cuando existe una diferenciación en la estructura de las profesiones: diferenciación del tipo de clientes, del local de trabajo, etc. De acuerdo con McGuire (1993), las transformaciones que afectan actualmente las profesiones son consecuencia de cinco factores: cambios en las características inherentes a cada profesión; transformaciones inherentes a la tecnología; cambios en las condiciones socio-económicas y en la cultura de la práctica profesional; cambios cualitativos en la rapidez, variedad y en las modalidades de comunicación; y cambios en las técnicas de manipulación de datos.

Scire. $9: 1$ (en.-jun. 2003) 85-93. 
Todo indica que van a surgir nuevos conceptos de organización de trabajo y de comportamiento con el desarrollo de las nuevas tecnologías de información, en función de su organización social y de las respuestas de los individuos que trabajan en los nuevos sistemas de información. Los profesionales de todos los campos del conocimiento se verán obligados a identificar las ventajas e inconvenientes de las aplicaciones de las tecnologías de información de forma que puedan optimizar los beneficios que pueden sacar de ellas para su práctica y para la sociedad.

De acuerdo con Friedson (apud Haug, 1973), los desarrollos socio-económicos que limitan la autonomía profesional engendrarían una "desprofesionalización". Haug define este fenómeno como la pérdida de las características principales de una profesión, particularmente de su monopolio sobre un conjunto de conocimientos, de la confianza del público, de su ética, de su autonomía y de su autoridad con relación a los clientes. De acuerdo con este autor, tal fenómeno de "desprofesionalización" ya está ocurriendo en todos los niveles de especialización a consecuencia de los cambios que se han producido en la división del trabajo, de la creación de nuevas actividades, de la informatización creciente y del desarrollo de las tecnologías de la información.

Sin duda, los cambios que ocurren en este campo están poniendo en cuestión los tres principios fundamentales de la teoría de las profesiones: el monopolio del conocimiento, la autonomía profesional y la orientación de servicio. Algunos factores han contribuido a la disminución de la autonomía profesional. Entre ellos se pueden citar la expansión de los conocimientos básicos y la modificación de las expectativas de los clientes. Además de eso, la información y las tecnologías de la información han desmitificado la dimensión de monopolio del conocimiento.

\section{Las profesiones de la información}

Desde la invención de prensa en el siglo XV hasta el presente, las profesiones de la información han vivido, según Starr (1996), un desafío y una transformación de una profundidad inigualable. Pero, a pesar de los cambios traídos por la historia, la misión fundamental de las profesiones de la información es - y continuará siendo - servir a la sociedad, respondiendo a sus necesidades de información; necesidades éstas, estables y permanentes.

Las transformaciones que ocurren en las profesiones de la información reflejan la evolución del sistema de las profesiones y apuntan, como afirma Cronin (1993) a cambios desde un núcleo tradicional, conocido y delimitado, hacia la periferia. Este autor afirma, a partir de la lectura de Abbott (1988) que los cambios en las profesiones ocurren siempre en los bordes de ese núcleo conocido y bien delimitado. Es exactamente en los límites de los bordes del núcleo de la

Scire. $9: 1$ (en.-jun. 2003) 85-93. 
profesión, donde se perciben las transformaciones. En este espacio es posible verificar, de forma más clara, los cambios que ocurren en las profesiones. Estos cambios, en el campo de las profesiones de la información, son el resultado de la diversidad de los soportes, de las funciones, de los roles, de los usos y de las expectativas de los usuarios en relación al tratamiento de la información. De hecho, la estructura de las unidades de información y los sectores de actividad donde la información es necesaria están cada vez más diversificados. En este sentido, las profesiones tradicionalmente vinculadas a la utilización, al análisis, y a la gestión de la información viven un momento incomparable. Por un lado, el aumento del uso de la información significa una gran diversificación en el trabajo de estos profesionales. Por otro lado, la "apropiación" del uso y de la gestión de la información por profesionales de otros campos del conocimiento significa, para muchos, una amenaza. Sin embargo, no nos parece que esta concurrencia represente una amenaza siempre que los profesionales de la información sepan aprovechar las oportunidades, utilizando sus habilidades y conocimientos y, sobretodo, transformándolos.

La explosión de la comunicación - consecuencia del desarrollo de la Internet y el incremento del valor de la información como recurso estratégico- están llevando cada vez más individuos a trabajar con fuentes de información. Este movimiento produce tensiones y nuevas oportunidades de alianzas en este espacio. El conjunto de estos movimientos, tensiones y alianzas esboza nuevos campos de competencia profesional, o nuevas "jurisdicciones profesionales" según el sentido que Abbott (1988) da a este término.

El futuro de las profesiones de la información está vinculado a su capacidad de adaptarse a estas nuevas demandas. Además de esto, esta diversidad en la forma de trabajar con la información demuestra una tendencia al trabajo interdisciplinario. En verdad, tener a la vez competencias en gestión, análisis y comunicación de la información y en tratamientos sofisticados para suministrar la información en red son tareas complejas que exigen, además de la especialidad en un tema determinado, el conocimiento de técnicas de comunicación, de animación, de informática y de gestión. Todo indica que los cambios tecnológicos y estructurales de la sociedad de información están obligando los profesionales de la información a redefinir su lugar en el mercado de trabajo. Al mismo tiempo que las funciones informativas se han tornado más complejas y específicas, esta tendencia a la especificidad no ha resultado en el fortalecimiento de las profesiones clásicas de la información (bibliotecarios, documentalistas y archiveros), pero ha abierto posibilidades a especialistas de otras disciplinas.

El desarrollo y la difusión de nuevos paradigmas tecnológicos, impulsado por la vinculación de las tecnologías electrónicas, ópticas, multimedia y de comunicación, han permitido una explosión informativa y comunicacional sin

Scire. $9: 1$ (en.-jun. 2003) 85-93. 
precedentes. Esta explosión ha incrementado y potenciado las capacidades de los profesionales de la información mediante la utilización de nuevas herramientas de búsqueda, de procesamiento, de almacenamiento, de acceso y de transferencia de la información. Por e contrario, el gran incremento en el uso de la información que ha resultado del desarrollo de la sociedad de la información la ha convertido en parte integrante del trabajo de un número cada vez mayor de profesionales como los gestores de la información, los especialistas de comunicación, los consultores, los educadores y los mediadores de información, entre otros. Como la información se ha convertido en el objeto principal de la actividad de gran parte de los individuos, la concepción del trabajo según la conocemos se está transformándos.

Nos parece importante citar algunos de los elementos que, además de las tecnologías, han tenido, según entendemos, una importancia fundamental en estas transformaciones: en primer lugar, el acceso individualizado e inmediato del usuario a la información; en segundo lugar, la fusión del contenido y de las nuevas tecnologías de mediación, que, como sucede en el caso del CD-ROM y de la Internet, proporcionan al usuario la posibilidad de acceder a la información sin intermediarios; en tercer lugar, la disminución creciente del costo de los aparatos y del acceso a la información; y, finalmente, el hecho de que productos, servicios y actividades de cualquier naturaleza sean cada vez más dependientes de la información que se renueva constantemente. (Cunha, 2000).

Según Couzinet (2001), el lugar de las profesiones de la información en la sociedad se desarrolla a través de un proceso lento. Sus competencias son cuestionadas cada vez que existe un adelanto en las tecnologías. Nuevos conceptos de organización del trabajo y de comportamiento surgen, en función del desarrollo de las nuevas tecnologías, de las nuevas organizaciones sociales y de las respuestas de los individuos que trabajan con nuevos sistemas de información.

Si es posible prever un rumbo en esta época de cambios, se puede afirmar que la evolución se orienta hacia nuevas alianzas, con el surgimiento de grupos interdisciplinarios; la confluencia de profesiones antes separadas, como, por ejemplo, el gestor de conocimiento - una mezcla de administrador, analista y bibliotecario - o el analista de la información en áreas específicas del conocimiento; la especialización por tipo de documento, como, por ejemplo, el administrador de sitios web; y nuevas responsabilidades, como, por ejemplo, el papel del mediador de información (Cunha, 2000). Estas transformaciones crean dificultades en la definición de los nuevos espacios de actuación profesional y también de convivencia de estos nuevos espacios con los tradicionales. Además de esto, es necesario recordar que el desarrollo de las nuevas tecnologías facilita la interpenetración entre las profesiones. Como resultado surgen nuevas categorías profesionales y las existentes se transforman. Según Almeida Jr. (2000), “casi siempre

Scire. $9: 1$ (en.-jun. 2003) 85-93. 
las discusiones sobre las funciones de los profesionales ocurren cerca de un hecho relevante - un nuevo siglo, una guerra, grandes alteraciones económicas... etc. [...] en estos momentos la sociedad entera es puesta en prueba y todas las áreas quedan obligadas a, como mínimo, cuestionarse sobre su presencia, función e importancia en el nuevo contexto".

Iniciamos un nuevo siglo, un tiempo de alteraciones económicas, sociales y políticas, un tiempo de perplejidades, de muchas preguntas y de pocas respuestas. Podemos preguntarnos, en este tiempo de perplejidades, si las profesiones de la información de la forma como las conocemos se mantendrán como están, qué transformaciones hay que prever y cómo preverlas.

La diversidad de situaciones que el profesional de la información enfrenta, la variedad de herramientas y de fuentes de información disponibles, la necesidad de participar, cada vez más, en proyectos interdisciplinarios, implican, a nuestro entender, la obligación de las escuelas de Biblioteconomía y Ciencia de la Información de abrir el campo de competencias para las que preparan. Esta apertura puede concretarse a través del establecimiento de cooperaciones con escuelas y facultades de otros campos del conocimiento. Como afirma Cubillo (1999) "debemos ser permeables a una influencia controlada de otras profesiones". En realidad, todas las profesiones tienen problemas con la gestión de la información y del conocimiento. Según este autor, la búsqueda de sinergias y de encuentros con otras áreas y otras profesiones puede ser enriquecedora para los profesionales de la información.

La evolución de las profesiones y la influencia que ejerce la globalización en todos los sectores de actividad han conducido a una alteración de la cultura de las profesiones vinculadas a la información y han hecho que los profesionales repiensen la naturaleza de su profesionalidad. En este sentido, las enseñanzas en Ciencia de la Información deben, según entendemos, habilitar al estudiante para que pueda reflexionar sobre la situación y, a partir de esta reflexión, responder a demandas de información cada vez más especializadas y diversificadas. En un ambiente en mutación permanente, la capacidad de aprender continuamente es esencial.

Para concluir, nos parece importante citar las palabras de Hobsbawn (1999) en la conclusión de su libro La era de los extremos:

No sabemos hacia dónde estamos yendo. Sólo sabemos que la historia nos trajo hasta este punto... Sin embargo, una cosa está clara. Si la humanidad quiere tener un futuro reconocible, no puede ser por el prolongamiento del pasado o del presente. Si intentamos construir el tercer milenio en esta base, vamos a fracasar. El precio del fracaso, - o sea, la alternativa para elcambio de la sociedad - es la oscuridad.

Scire. $9: 1$ (en.-jun. 2003) 85-93. 


\section{Referencias}

Abbott, A. (1988). The system of professions: an essay on the division of expert labour. Chicago: The University of Chicago, 1988.

Almeida, J. R. O. F. (2000). Profissional da informação: entre o espírito e a produção. // Valentim, M. O Profissional da Informação: formação, perfil e atuação profissional. São Paulo: Editora Polis/APB, 2000.

Bennett JR., W. S.; Hokenstad JR, M. C. (1973). Full-time people workers and conceptions of the "professional". // The Society Review Monograph. 20 (Dec.1973) 21-43.

Cronin, B. (1983). Post-industrial society: some manpower issues for the library information profession. // Journal of Information Science. 7 (1983) 1-14.

Couzinet, V. (2001). Organisation et dispositif d'intéressement: la professionnalisation des documentalistes. // Séminaire 2000-2001. Toulouse: LERASS, 2001.

Cubillo, J. (1999). El profesional sin nombre: reflexiones sobre uma reflexión. // Comunicado CEPAL/CLADES, (nov.1999).

Cunha, M. V. (2000). O profissional da informação e o mercado de trabalho. // Informação \& Sociedade. 10:1 (jan./jun.2000) 159-167.

Curry, L.; Wergin, J.F. (1993). Setting priorities for change in professional education. // Educating professionals. San Francisco: Jossey-Bass, 1993. 316-327.

Haug, M. R. (1973). Deprofessionalization: an alternate hypothesis for the future. // The Society Review Monograph. 20 (Dec.1973) 195-211.

Hobsbawn, E. (1999). A era dos extremos: o breve século XX, 1919-1991. 2.ed. São Paulo: Companhia das Letras, 1999.

McGuire, C. (1993). Socio-cultural changes affecting professions and professionals. // Curry, L.; Wergin, J. F. Educating professionals. San Francisco: Jossey-Bass, 1993. 3-16.

Maceviciuté, E. (1998). Survey of the labour market for information specialists in Lithuania. // Information Research. 3:4 (Apr.1998).

Starr, K. (1996). Task force on the future of Librarianship. Los Angeles, Ca.: The Library Association, 1996.

Scire. $9: 1$ (en.-jun. 2003) 85-93. 